\title{
Compressed UAV sensing for flood monitoring by solving the continuous travelling salesman problem over hyperspectral maps
}

Pablo Casaseca-de-la-Higuera, Antonio Tristán-Vega, Carlos Hoyos-Barceló, Susana Merino-Caviedes, Qi Wang, et al.

Pablo Casaseca-de-la-Higuera, Antonio Tristán-Vega, Carlos HoyosBarceló, Susana Merino-Caviedes, Qi Wang, Chunbo Luo, Xinheng Wang, Zhi Wang, "Compressed UAV sensing for flood monitoring by solving the continuous travelling salesman problem over hyperspectral maps," Proc. SPIE 10784, Remote Sensing of the Ocean, Sea Ice, Coastal Waters, and Large Water Regions 2018, 107840D (10 October 2018); doi:

$10.1117 / 12.2325645$

SPIE. Event: SPIE Remote Sensing, 2018, Berlin, Germany 


\title{
Compressed UAV sensing for flood monitoring by solving the continuous travelling salesman problem over hyperspectral maps
}

\author{
Pablo Casaseca-de-la-Higuera ${ }^{\mathrm{a}, \mathrm{c}}$, Antonio Tristán-Vega ${ }^{\mathrm{b}}$, Carlos Hoyos-Barcelóc, Susana \\ Merino-Caviedes ${ }^{\mathrm{a}}$, Qi Wang ${ }^{\mathrm{c}}$, Chunbo Luo ${ }^{\mathrm{d}}$, Xinheng Wang ${ }^{\mathrm{e}}$, and Zhi Wang ${ }^{\mathrm{f}}$ \\ ${ }^{a}$ Laboratorio de Procesado de Imagen. ETSI de Telecomunicación, Universidad de Valladolid. \\ Valladolid, 47011, Spain \\ ${ }^{\text {b}}$ Departamento de Ingeniería Mecánica. Escuela de Ingenierías Industriales, Universidad de \\ Valladolid. Valladolid, 47011, Spain \\ ${ }^{\mathrm{C}}$ Centre for Artificial Intelligence, Visual Communications, and Networks, School of \\ Computing, Engineering and Physical Sciences, University of the West of Scotland, Paisley \\ PA1 2BE, United Kingdom \\ ${ }^{\mathrm{d}}$ Department of Computer Science, College of Engineering, Mathematics and Physical \\ Sciences, University of Exeter, Exeter EX4 4QJ, United Kingdom \\ ${ }^{e}$ School of Computing and Engineering. University of West London, London W5 5RF, United \\ Kingdom

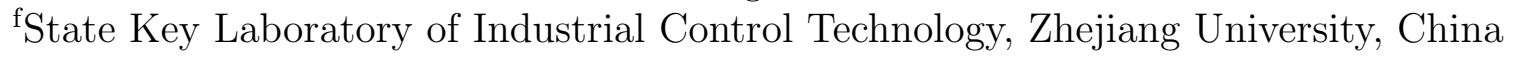

\begin{abstract}
Unmanned Aerial Vehicles (UAVs) have shown great capability for disaster management due to their fast speed, automated deployment and low maintenance requirements. In recent years, disasters such as flooding are having increasingly damaging societal and environmental effects. To reduce their impact, real-time and reliable flood monitoring and prevention strategies are required. However, the limited battery life of small lightweight UAVs imposes efficient strategies to subsample the sensing field in this context. This paper proposes a novel solution to maximise the number of inspected flooded surface while keeping the travelled distance bounded. Our proposal solves the so-called continuous Travelling Salesman Problem (TSP), where the costs of travelling from one location to another depend not only on the distance, but also on the presence of water. To determine the optimal path between checkpoints, we employ the fast sweeping algorithm using a cost function defined from hyperspectral satellite maps identifying flooded regions. Preliminary results using MODIS flood maps show that our UAV planning strategy achieves a covered flooded surface approximately 3.33 times greater for the same travelled distance when compared to the conventional TSP solution. These results show new insights on the use of hyperspectral imagery acquired from UAVs to monitor water resources.
\end{abstract}

Keywords: Flood Monitoring, UAV, Optimal Path Planning, Hyperspectral Imaging.

\section{INTRODUCTION}

In recent years, disasters such as flooding are having increasingly damaging societal and environmental effects. ${ }^{1}$ To reduce the impact of flooding, real-time and reliable flooding monitoring strategies are required. The monitoring of flash floods poses practical difficulties to conventional monitoring networks. While satellite images provide valuable information of flooded areas, those observations get obscured by the extensive mantle of clouds that forms on those events. ${ }^{2}$ Moreover, the high operating costs of aerial monitoring when paired with the high volubility in time and location of these sparse events has forced the process to be more reactive than active, and due to the time it takes to mobilize the monitoring resources, and the transient nature of this phenomena, there is little data on the processes concurrent and previous to extreme flash floods. ${ }^{3}$

Corresponding author: P. Casaseca-de-la-Higuera (Email: casaseca@lpi.tel.uva.es, Telephone: +34 983 185591)

Remote Sensing of the Ocean, Sea Ice, Coastal Waters, and Large Water Regions 2018, edited by Charles R. Bostater, Stelios P. Mertikas, Xavier Neyt, Proc. of SPIE Vol. 10784,

107840D · @ 2018 SPIE · CCC code: 0277-786X/18/\$18 · doi: 10.1117/12.2325645 
The use of small Unmanned Aerial Vehicles (UAVs) in recent times has enabled geographic surveillance in dangerous or previously inaccessible locations, while simultaneously reducing the operating costs and the response time. ${ }^{4}$ UAVs have shown great capability in military and civilian areas and attracted enormous interests from both academia and industry worldwide. The UAV market was valued at USD 18.14 Billion in 2017 and is projected to reach USD 52.30 Billion by $2025 .{ }^{5}$ UAVs have the advantages of fast speed, automated deployment and low maintenance requirements, thus making them suitable for a wide range of commercial and research areas such as disaster management including flood monitoring and prevention. Inexpensive, low-altitude multicopter UAVs equipped with state-of-the-art monitoring technology feature a variety of sensors that can be used to identify environmental hazards, help in the detection of missing people, and leverage communication networks in contexts where such communications are compromised due to a natural disaster.

Monitoring with UAVs is performed by setting landmarks or checkpoints for the UAV to visit and take measurements. Abdelkader et al. ${ }^{6}$ proposed a system for real-time flood monitoring where UAVs drop buoyant Lagrangian micro-sensors in selected locations, which transmit information as they are carried away by the flood. In addition, UAVs equipped with on-board cameras can be deployed to provide close on-site monitoring of potentially flooded or damaged areas. This can range from the use of conventional video recording or thermal imaging to search missing people or assess the impact or damage to more involved remote hyperspectral sensing to detect and identify the potential damage. Finally, in emergency missions, UAVs can be used to enable communication links when network nodes have been damaged. The UAVs would be deployed as relay nodes and become part of the network so as to guarantee stable communication for emergency services.

The use of UAV technology allows for real-time tracking, constituting a significant improvement in time resolution compared to satellite imagery (approximately 1 hour vs. 1-3 days depending on surveillance area and flying altitude of UAV). There is however a major restriction when using small lightweight UAVs for remote flood monitoring and prevention. Their limited battery life and on-board computation capability constitute important challenges for their deployment in real-time monitoring scenarios. These challenges impose the need to optimise the amount of monitored surface for each UAV mission before batteries drain.

There is thus need of efficient strategies to subsample the sensing field, hereinafter considered as a twodimensional grid of cells whose size is the drones field of view. A compressed sensing strategy where a reduced number of checkpoints is defined in flooded areas, constitutes a sensible approach in this context. The UAV would need to visit each cell from its starting point and return back to charge or replace batteries. Determining the shortest-path route can be easily achieved by solving the well-known travelling salesman problem (TSP). ${ }^{7}$ To solve this problem, the collection of checkpoints and pair-wise travel costs (distances) is fed into an optimization algorithm to obtain the optimal route. During the trips, the drone will fly over some additional flooded areas, thus inspecting a higher number of flooded cells than the number of checkpoints. This approach is however suboptimal, since there is no control on how much intermediate flooded cells will be inspected.

This paper proposes a novel solution to maximise the number of inspected flooded cells while keeping the travelled distance bounded. Our proposal solves the so-called continuous TSP, ${ }^{8}$ where the costs of travelling from one cell to another depend not only on the distance, but also on the presence of water therein. To determine the optimal path between checkpoints, we employ the fast sweeping algorithm using a cost function defined from hyperspectral satellite maps identifying flooded regions. This water-informed cost is then used as the distance function to solve the TSP and provide the final UAV route.

\section{PROBLEM STATEMENT}

We start by considering a rectangular 2D map where the initial surveillance is planned. The map is divided in $M \times N$ cells for inspection where the area of each cell corresponds to the field of view of the drone's camera from its flying altitude. The maps are processed in such a way that each cell corresponds to an image pixel.

The problem is stated so that the following question is solved: "Given some prior knowledge of the field of interest, how should UAVs move to gather maximum information, subject to energy and other constraints?". The most common scenario only considers energy constraints, and the problem is solved so that the flown distance is minimised. In a more complex scenario such as the one at hand, we want to introduce a trade-off between the drone's travelled distance and the amount of flooded surface covered by the drone. 
The prior knowledge of flooded areas can be gathered from previously processed hyperspectral maps. In this paper, we have used initial flood maps obtained from MODIS hyperspectral data ${ }^{9}$ Figure 1 shows the employed MODIS hyperspectral flood maps where flooded areas and watercourses are identified in different colours. An alternative approach would be to use automatically classified hyperspectral maps obtained from the actual UAV at higher altitudes prior to close inspection.

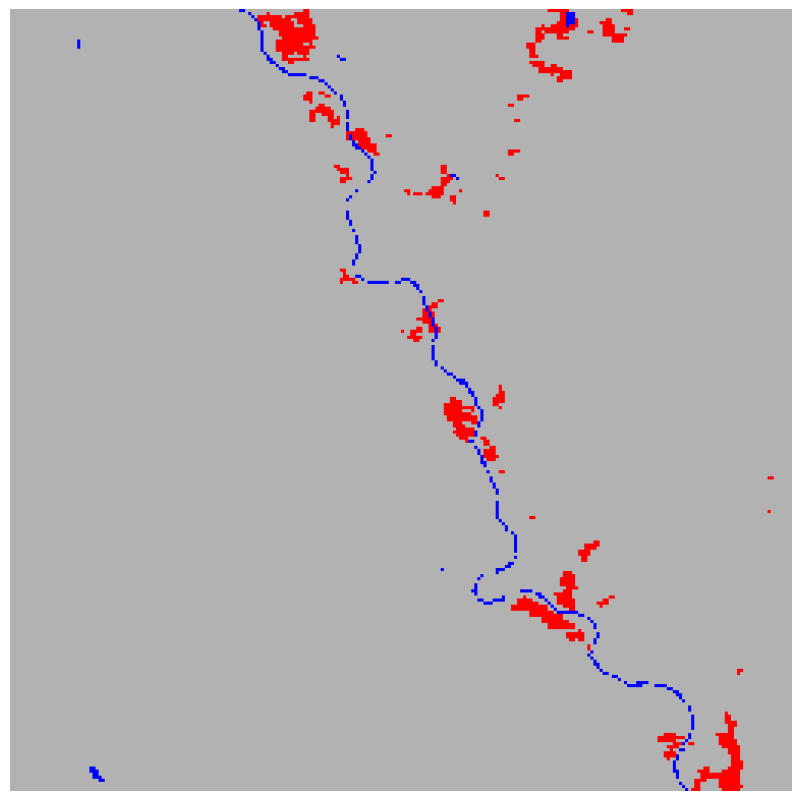

Figure 1: Region from MODIS hyperspectral flood map ${ }^{9}$ employed in this paper. Flooded areas are shown in red, and watercourses in blue. Gray regions corresponde to dry or cloudy areas.

As a starting point, a set of $K$ visiting points are randomly chosen in the flooded areas following a compressed sensing phylosophy, and the problem is stated as follows: Given a departure point for the UAV, calculate the optimal path so that all $K$ points are visited with the best trade-off between travelled instance, and the amount of covered flooded surface. This problem resembles the well-known TSP: given a collection of cities, and a cost of travelling from one city to the next, find a way to travel to all the cities with the minimal total cost. The TSP is typically posed as follows: starting from a set of cities and a collection of links between the cities, each link carries a cost of travelling between the two cities, which is often the distance, or the time spent. Then, a dynamic optimization problem is solved to obtain the optimal solution, which scales in complexity when the number of cities (points) is high. However, there are heuristic algorithms ${ }^{10-12}$ which efficiently find sub-optimal solutions.

We are instead interested in a continuous version of this problem. In our problem, we are considering the amount of covered flooded surface, apart from the travelled distance. So, in principle, we do not know what the cost of travelling between the points will be. This can be modelled as a cost function $M(\mathbf{x})$, where the cost of travelling from one point to the next depends on the position in the map $\mathbf{x}=(x, y)$. Then, we do not even know the optimal path between two points, which, may indeed not be a straight line if flooded areas are considered as well. The straight line would be the optimal if a constant cost were considered. This is the case where only distance or time are used to define those costs.

Then, the hyperspectral maps are used to define this cost function $M(\mathbf{x})$ where watercourses and flooded areas are assigned different costs so that the preferable regions are the flooded ones. By using space-varying costs, the problem solution provides a trade-off between the actual travelled distance and the amount of flooded 
surface covered by the drone. In this paper, we have defined $M(\mathbf{x})$ as follows:

$$
M(\mathbf{x})=d(\mathbf{x})-\alpha f(\mathbf{x})-\beta w(\mathbf{x})
$$

where $d(\mathbf{x})=1$ is a constant mask so that it accounts for distance, and $f(\mathbf{x})$ and $w(\mathbf{x})$ are image masks that respectively represent the presence $(1)$ or absence $(0)$ of flooded areas $(f)$ or watercourses $(w)$. The masks used to construct $M(\mathbf{x})$ from Figure 1 are represented in Figure 2.

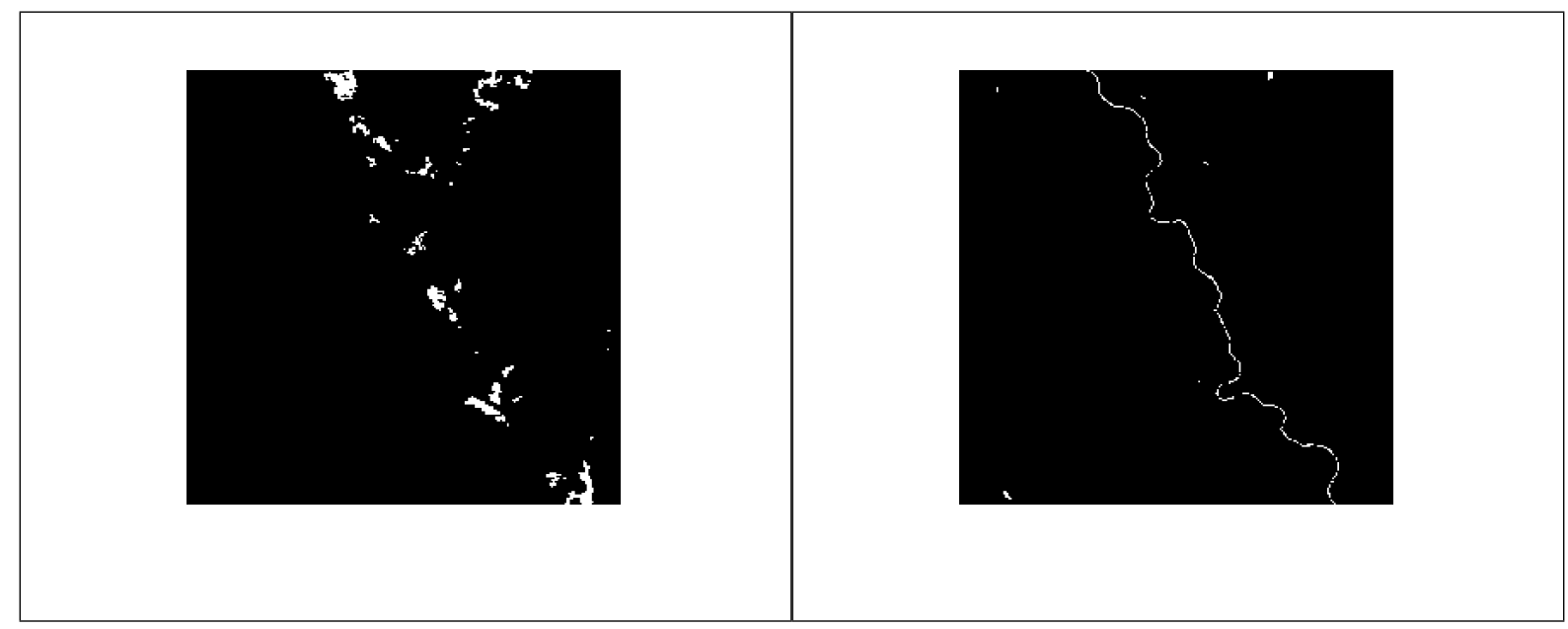

(a) $f(\mathbf{x})$

(b) $w(\mathbf{x})$

Figure 2: Flood $f(\mathbf{x})$ and watercourse $w(\mathbf{x})$ masks used to define the cost function $M(\mathbf{x})$.

The values $\alpha=0.9$ and $\beta=0.3$ have been chosen so that the lower cost (0.1) is assigned to flooded areas, and the higher (1) to dry/undefined regions. Watercourses are assigned an intermediate cost (0.7). In principle, they are already known to be there, and may not need to be covered. However, it is preferable to cover them than dry areas due to their potential proximity to flooded areas. On the other hand, in some situations (e.g., when looking for missing people), they may need to be assigned an even lower cost. Figure 3 shows the overall cost function $M(\mathbf{x})$ corresponding to Figure 1.

\section{METHODOLOGY}

The continuous TSP can be solved by embedding the solution of boundary problems of the Eikonal equation inside an algorithm to solve the conventional, discrete TSP. The Eikonal equation is a non-linear partial differential equation usually found in front propagation problems which actually provides the shortest path between two points on a surface or volume, i.e., the geodesic curve. The formulation of the Eikonal equation is:

$$
\|\nabla u(\mathbf{x})\|=\frac{1}{f(\mathbf{x})}, \mathbf{x} \in \Omega
$$

subject to $\left.u\right|_{\partial \Omega}=0$, where $\Omega$ is an open set in $\mathbb{R}^{n}$ with well-behaved boundary, $f(\mathbf{x})$ is a function with positive values, $\nabla$ denotes the gradient, and $\|\cdot\|$ the Euclidean norm. The physical interpretation of the solution is that $u(\mathbf{x})$ represents the shortest time to travel from $\partial \Omega$ to a point $\mathbf{x} \in \Omega$, with $f(x)$ the velocity at that point. Note that Equation (1) can be embedded in (2), as the cost function can be considered proportional to the inverse of the speed function

$$
M(\mathbf{x}) \propto \frac{1}{f(\mathbf{x})}
$$

The proposed method to solve the continuous TSP for the problem at hand is: 


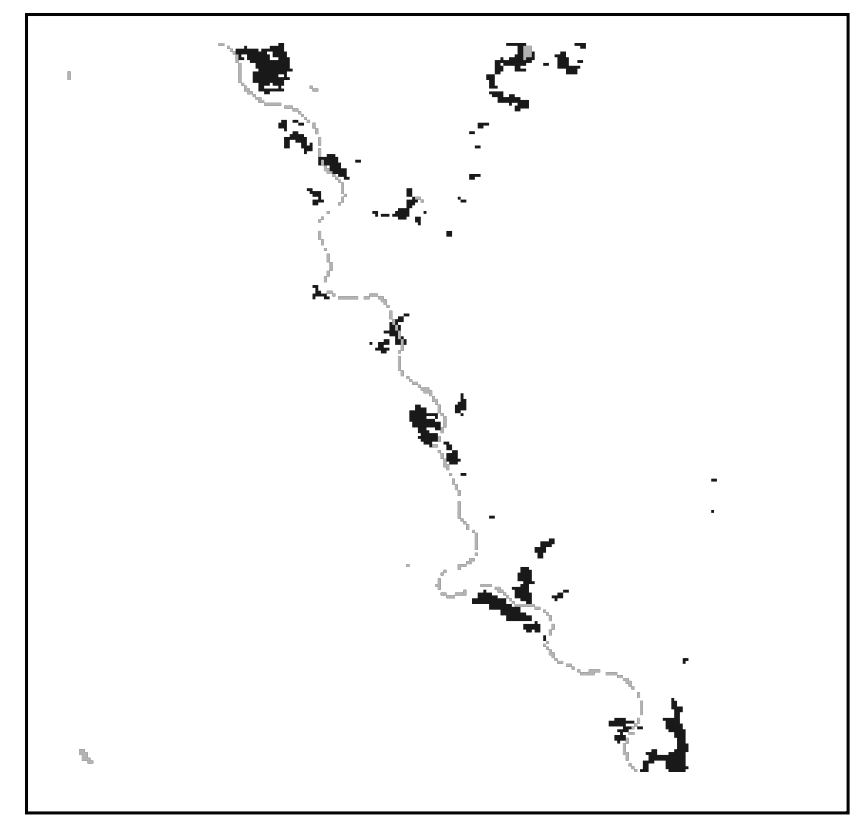

Figure 3: Representation of the cost function $M(\mathbf{x})$ constructed from the hyperspectral map in Figure 1.

1. Specify $K$ points at different flooded locations in the map.

2. Pick one of the $K+1$ points ( $K$ plus UAV departure point), and compute the shortest geodesic from every other point back to it solving the Eikonal equation. To do so, we employ the optimization strategy proposed in $^{13}$ based on the fast sweeping algorithm introduced by Kao et al. ${ }^{14}$ Our source code ${ }^{*}$ implements the multi-threaded fast-sweeping suggested by Zhao ${ }^{15}$ and it is programmed in C++/ITK. Fast sweeping is based on dynamic programming, and guarantees the convergence of iterative local computations to the globally shortest geodesic.

3. At this point, all the costs and paths between all pairs of points are available. These are passed to a MATLAB solver based on integer-linear programming to provide the optimal solution to the discrete TSP problem.

\section{RESULTS AND DISCUSSION}

We used the map in Figure 1 to carry out experiments for $K=\{3,10,20,50\}$. They respectively correspond to $0.04 \%, 0.12 \%, 0.24 \%$, and $0.59 \%$, of the flooded surface. The percentage of covered flooded surface after running the algorithm is presented in table 2 and compared to the one achieved by solving the discrete TSP where the shortest path between points is chosen in terms of distance (i.e., $M(\mathbf{x})=1$ in Equation (1)). Table 1 shows the corresponding UAV travelled distances obtained with both planning methods. Distances have been normalized to the side of one square cell.

A joint analysis of both tables leads to the following conclusions:

- The proposed path planning strategy achieves a larger amount of covered surface than using discrete TSP planning. Table 2 shows that only 3 visits are needed to cover $9 \%$ of the overall flooded surface. To achieve the same covered surface, the UAV needs to visit 50 landmarks. This means that our proposal manages to raise the covered flooded surface from $0.04 \%$ to $9 \%$ whereas the discrete TSP needs to start at $0.59 \%$. When performing 50 visits, the proposed strategy almost doubles the covered surface, achieving $16 \%$.

\footnotetext{
* Our code is available at https://www.nitrc.org/projects/riemantract.
} 
Table 1: Overall flooded covered surface with the proposed method compared with discrete TSP.

\begin{tabular}{|c|c|c|c|}
\hline$K$ & $\begin{array}{c}\text { Initial flooded surface } \\
\mathbf{( \% )}\end{array}$ & $\begin{array}{c}\text { Final covered flooded surface } \\
\text { with our method (\%) }\end{array}$ & $\begin{array}{c}\text { Final covered flooded surface } \\
\text { with discrete TSP (\%) }\end{array}$ \\
\hline 3 & $0.04 \%$ & $9 \%$ & $1 \%$ \\
\hline 10 & $0.12 \%$ & $10 \%$ & $2 \%$ \\
\hline 20 & $0.24 \%$ & $11 \%$ & $3 \%$ \\
\hline 50 & $0.59 \%$ & $16 \%$ & $9 \%$ \\
\hline
\end{tabular}

Table 2: Overall UAV travelled distance obtained with the proposed method compared with discrete TSP.

\begin{tabular}{|c|c|c|}
\hline$K$ & $\begin{array}{c}\text { Normalized travelled distance with our } \\
\text { method }\end{array}$ & $\begin{array}{c}\text { Normalized travelled distance with } \\
\text { discrete TSP }\end{array}$ \\
\hline 3 & 2,467 & 1,978 \\
\hline 10 & 2,635 & 2,185 \\
\hline 20 & 3,073 & 2,591 \\
\hline 50 & 4,266 & 3,770 \\
\hline
\end{tabular}

- The travelled distance to cover the same amount of flooded surface is significantly lower for our proposal. For instance, to cover $9 \%$ of the surface, the UAV would need to travel for 2,467 normalised units with our method, which would raise to $3,770(\approx 50 \%$ increase $)$ using discrete TSP planning.

- Our UAV planning strategy achieves a covered flooded surface approximately 4 times greater for the same travelled distance when compared to the conventional TSP solution. Looking at comparable figures in Table 1, our methods yields a travelled distance for the UAV of 2, 467 normalised units when visiting 10 points. On the other hand, path planning using conventional TSP would yield 2,591. The amount of covered surface $10 \%$ for our method and $3 \%$ with conventional TSP, leading to a $3.33 \times$ improvement.

Figure 4 shows the optimal paths obtained with both approaches for $K=10$ initial visiting points. It can be obseved that paths obtained with the proposed method tend to pass over flooded and water areas more than those obtained with discrete TSP do.

\section{CONCLUSION AND FUTURE LINES}

We have proposed a novel UAV path planning methodology to monitor flooding episodes that maximises the covered flooded areas while keeping the total travel distance bounded. Our proposal solves the continuous TSP, where the costs of travelling from one cell to another depend not only on the distance, but also on the presence of water therein. To determine the optimal path between checkpoints, we employ the fast sweeping algorithm using a cost function defined from hyperspectral satellite maps identifying flooded regions. This water-informed cost is then used as the distance function to solve the TSP and provide the final UAV route.

Results show that the proposed UAV path planning strategy achieves a covered flooded surface approximately 4 times greater for the same travelled distance when compared to the conventional TSP solution. In addition, to achieve the same covered surface, the path provided by the discrete TSP solution would be $50 \%$ longer than ours. These results show new insights on the use of hyperspectral imagery acquired from UAVs to monitor water resources.

Our preliminary results show new insights on the use of hyperspectral imagery acquired from UAVs to monitor water resources. We are currently working on improving our method to make it fully deployable in a real environment. The points that are being explored as further lines of this work are:

- Optimal positioning of visiting points within flooded areas instead of random spread. 


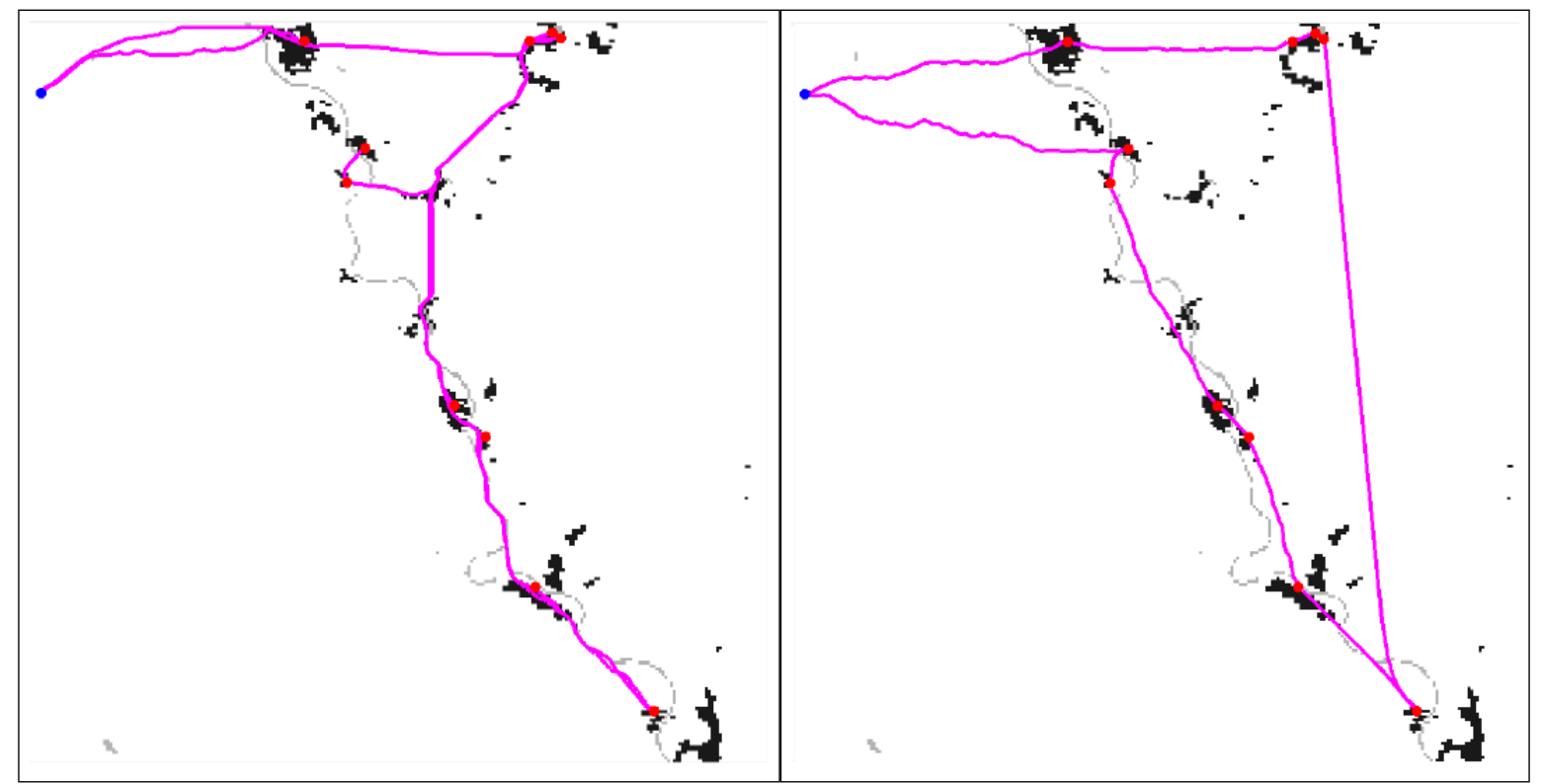

(a) Our method

(b) Discrete TSP

Figure 4: Optimal path planning obtained for $K=10$ with the proposed method and the conventional, discrete TSP.

- Include directional costs in the cost function $M(\mathbf{x})$. One of the reasons for choosing fast sweeping instead of more widely employed algorithms such as fast marching, ${ }^{16}$ is the possibility of including anisotropy in the definition of cost functions. This would help for instance in the design of optimal paths that follow a specific shape of a flooded area region or watercourse profile. The definition of anisotropic cost functions is considered as a future line for this research.

- Real embedding of the fast sweeping algorithm into the problem. The calculation of optimal paths in this paper was sub-optimal since not all the pair-wise paths need to be computed as many will not be taken. Another future line is to embed the fast sweeping algorithm into the TSP algorithm itself.

- Include drone dynamics into the planning equations, which so far has not been carried out.

\section{Acknowledgments}

This work was funded by the Royal Society of Edinburgh and National Science Foundation of China within the international project "Flood Detection and Monitoring using Hyperspectral Remote Sensing from Unmanned Aerial Vehicles" (project NNS/INT 15-16 Casaseca).

\section{REFERENCES}

[1] of Transport Economics, B., "Economic costs of natural disasters in australia, report 103," (2001).

[2] Feng, Q., Liu, J., and Gong, J., "Urban flood mapping based on unmanned aerial vehicle remote sensing and random forest classifiera case of yuyao, china," 7, 1437-1455 (03 2015).

[3] Perks, M. T., Russell, A. J., and Large, A. R. G., "Technical note: Advances in flash flood monitoring using unmanned aerial vehicles (uavs)," Hydrology and Earth System Sciences 20(10), 4005-4015 (2016).

[4] Fekete, B. M., Robarts, R. D., Kumagai, M., Nachtnebel, H.-P., Odada, E., and Zhulidov, A. V., "Time for in situ renaissance," Science 349(6249), 685-686 (2015).

[5] "Unmanned Aerial Vehicle market. analysis and forecast to 2025." https: //www . marketsandmarkets . com/ PressReleases/unmanned-aerial-vehicles-uav.asp. Accessed: 23/07/2018. 
[6] Abdelkader, M., Shaqura, M., Claudel, C. G., and Gueaieb, W., "A uav based system for real time flash flood monitoring in desert environments using lagrangian microsensors," in [2013 International Conference on Unmanned Aircraft Systems (ICUAS)], 25-34 (May 2013).

[7] Arora, S., "Polynomial time approximation schemes for euclidean traveling salesman and other geometric problems," J. ACM 45, 753-782 (Sept. 1998).

[8] Sahai, T., Klus, S., and Dellnitz, M., "Continuous relaxations for the traveling salesman problem," CoRR abs/1702.05224 (2017).

[9] Carroll, M. L., DiMiceli, C. M., Townshend, J., Sohlberg, R. A., and Noojipady, P., "Mod_flood.2008165.midwest.geog," in [MODIS Flood Map. 2008 US Midwest Flood,11-13 2008], Carroll, M. L., DiMiceli, C. M., Townshend, J., Sohlberg, R. A., and Noojipady, P., eds., University of Maryland (2008).

[10] Wei, J.-D. and Lee, D. T., "A new approach to the traveling salesman problem using genetic algorithms with priority encoding," in [Proceedings of the 2004 Congress on Evolutionary Computation (IEEE Cat. No.04TH8753)], 2, 1457-1464 Vol.2 (2004).

[11] Chen, J., Ye, F., and Li, Y., "Travelling salesman problem for uav path planning with two parallel optimization algorithms," in [2017 Progress in Electromagnetics Research Symposium - Fall (PIERS - FALL)], $832-837$ (2017).

[12] Syambas, N. R., Salsabila, S., and Suranegara, G. M., "Fast heuristic algorithm for travelling salesman problem," in [2017 11th International Conference on Telecommunication Systems Services and Applications (TSSA)], 1-5 (2017).

[13] Melonakos, J., Pichon, E., Angenent, S., and Tannenbaum, A., "Finsler active contours," IEEE Transactions on Pattern Analysis and Machine Intelligence 30(3), 412-423 (2008).

[14] Kao, C. Y., Osher, S., and Qian, J., "Laxfriedrichs sweeping scheme for static hamiltonjacobi equations," Journal of Computational Physics 196(1), 367 - 391 (2004).

[15] Zhao, H., "Parallel implementations of the fast sweeping method," J. Comput. Methods 25(4), $421-429$ (2007).

[16] Sethian, J. A., "A fast marching level set method for monotonically advancing fronts," Proceedings of the National Academy of Sciences 93(4), 1591-1595 (1996). 